\title{
Develop your business with Virtual reality organization
}

\author{
Hiverlab Pte $\operatorname{Ltd}^{1}$ \\ ${ }^{1}$ Affiliation not available
}

April 29, 2020

Virtual reality, the idealized next step in both gaming and simulations. With the rise of Oculus Rift, HTC Vive, Sony's Project Morpheus and the recent One plus Cardboard virtual reality is beginning to find a place in the consumer household, rather than with only arcades and technology enthusiasts.

Such massive market potential is likely the driving force behind the broad range of companies jumping on the VR scene. Everyone from Sony to Valve are currently developing or have released early products, such a range of heavy hitting gaming companies is a sign that Virtual Reality Company Singaporehas a place. Whilst this anticipated growth is impressive, it remains to be seen if this new technology will be purely a passing fad or a long-term gaming option.

According to this report the constant increase in technology, such as $3 \mathrm{D}$ effects and motion tracking are pushing the rise of virtual reality. Whilst increasing disposable incomes are letting more and more households give this new technology a try. On the flip-side the cost of these technologies, demonstrated by Valve stating that their HTC Vive is aimed at the high-end consumer is expected to harm growth and widespread adoption.

The worldwide spread of VR is obviously concentrated within North America and Europe, with both representing $69 \%$ of the revenue share. Though surprisingly Asia is slated as the region of highest potential due to the growing online involvement and presence of Sony, who have easy to Asia for their Project Morpheus.

This single report highlights that VR has huge potential, both for the companies producing them and the consumers. Yet ultimately it will depend on the content that will be available for these machines and the cost of them. At the current time an average consumer will not spend the equivalent of an Xbox One or PlayStation 4 for a machine that offers only a limited range of games. Though if games such as the "Keep Talking and Nobody Explodes" demonstrate anything, it is that the right sort of game style can work seamlessly with headset VR. We look forward to the day that Call of Duty can be played on a Best Virtual Reality Companiesmachine, or maybe that wouldn't be such a good idea.

Source URL : - https://penzu.com/p/0fa011fc

For more info : - Best Augmented Reality Companies 